\title{
Adaptive Beamforming Technique for Accurate Vertical Wind Measurements with Multichannel MST Radar
}

\author{
KOJI NISHIMURA \\ Research Organization of Information and Systems, Transdisciplinary Research Integration Center, \\ Tachikawa, Japan \\ TAKUJI NAKAMURA \\ National Institute of Polar Research, Tokyo, Japan \\ TORU SATO \\ Department of Communications and Computer Engineering, Kyoto University, Kyoto, Japan \\ KAORU SATO \\ Department of Earth and Planetary Science, The University of Tokyo, Tokyo, Japan
}

(Manuscript received 20 April 2011, in final form 25 July 2011)

\begin{abstract}
Aspect-sensitive backscattering of the atmosphere causes a small error in an effective line-of-sight direction in vertical beam observations leading to a serious degradation of vertical wind estimates due to contamination by horizontal wind components. An adaptive beamforming technique for a multichannel mesosphere-stratospheretroposphere (MST) radar is presented, which makes it possible to measure the vertical wind velocity with higher accuracy by adaptively generating a countersteered reception beam against an off-vertically shifted echo pattern. The technique employs the norm-constrained direction-constrained minimization of power (NC-DCMP) algorithm, which provides not only robustness but also higher accuracy than the basic direction-constrained minimization of power algorithm in realistic conditions. Although the technique decreases the signal-to-noise ratio, the ratio is controlled and bound at a specified level by the norm constraint. In the case that a decrease of $-3 \mathrm{~dB}$ is acceptable in a vertical beam observation, for which usually a much higher signal-to-noise ratio is obtained than for oblique beams, the maximum contamination is suppressed to $1 / 10$ even for the most imbalanced aspect sensitivity.
\end{abstract}

\section{Introduction}

Accurate measurements of the vertical wind velocity are important in understanding the dynamics of atmospheric phenomena having various spatial scales, vertical coupling in the atmosphere through momentum transport by internal waves, and driving mechanisms of global circulation. In the troposphere, vertical wind fluctuations play an important role in the vertical transport of heat and momentum associated with severe atmospheric disturbances such as typhoons (Sato et al. 1991; Sato 1993). Ageostrophic circulations including vertical motions

Corresponding author address: Koji Nishimura, National Institute of Polar Research, Tachikawa, Tokyo 1908518, Japan.

E-mail: knish@ieee.org maintain the geostrophic and hydrostatic structure of synoptic-scale atmospheric phenomena such as jet streaks (Takayabu et al. 2000).

In the middle atmosphere, the vertical transport of horizontal momentum by atmospheric waves such as Rossby waves and gravity waves is essential to drive material circulations (e.g., Plumb 2002; McLandress and Shepherd 2009; Okamoto et al. 2011). The material circulation not only globally transports minor constituents such as ozone but also causes significant departure of the thermal structure from a simple radiative balance through adiabatic heating and cooling associated with its vertical motion. Temperature perturbations caused by vertical fluctuations associated with gravity waves largely affect the amounts of unique clouds in the polar middle atmosphere such as polar stratospheric and mesospheric 
clouds (Eckermann et al. 2009; Alexander et al. 2011; Kohma and Sato 2011; Taylor et al. 2011).

Mesosphere-stratosphere-troposphere (MST) radar, which observes a wide altitude range of the neutral atmosphere, is an indispensable instrument because of its ability to directly estimate vertical Doppler velocities from atmospheric echoes. It is well known that, in veryhigh frequency (VHF) radar observations, aspectsensitive scattering exists and strongly affects the echo distribution when the radar beam is near vertical (Gage and Green 1978; Röttger 1980). This aspect sensitivity is evident uniquely in VHF bands and is considered a direct consequence of spectral enhancement, which is possibly caused by a horizontal layered structure of the refractive index (Tsuda et al. 1986; Worthington et al. 1999). Potential mechanisms have been proposed to explain the creation of the structure: those relating to Kelvin-Helmholtz instability (Muschinski 1996) and those relating to gravity waves (Nastrom and VanZandt 1994; Tsuda et al. 1997). Aspect sensitivity becomes more complex when the laminated structure is not horizontal because of modification by small horizontal-scale mountain waves (Sato 1990).

By these mechanisms, the brightest direction is tilted slightly off-vertical, leading to contamination of the horizontal wind components to the "vertical" Doppler velocity estimate. Even if this unexpected off-vertical angle is small, often the horizontal components are large enough to smear the faint vertical component. For example, for the vertical beam with $1^{\circ}$ from the zenith, a horizontal velocity of $30 \mathrm{~m} \mathrm{~s}^{-1}$ discharges a Doppler velocity of $0.52 \mathrm{~m} \mathrm{~s}^{-1}$, which is usually unacceptable for vertical wind measurement. Even in discussing local dynamics, the error should be less than $0.1 \mathrm{~m} \mathrm{~s}^{-1}$.

Fukao et al. (1991) discussed a systematic reversal of the vertical wind at the height where the horizontal wind velocity peaks. There are several possible physical mechanisms to explain the systematic structure. Muschinski (1996) explained it as a consequence of Kelvin-Helmholtz instabilities. Takayabu et al. (2000) interpreted it as secondary circulation of jet streaks. Sato (1990) showed that the time-mean vertical profile of vertical winds is reflected by the structure of quasi-stationary topographically forced gravity waves. However, for quantitative discussion of these mechanisms, we need to reduce errors due to aspect sensitivity by having a target volume as small as possible.

Recently, MST radar instrumentation has been updated or newly constructed with multichannel digital receiver systems that are capable of receiving signals at each segment of the antenna array so that additional information about the structure within a main beam can be observed (Hassenpflug et al. 2008).
Palmer et al. (1991) discussed measurement of the vertical velocity under an aspect-sensitive condition by means of a spatial antenna method using a multichannel middle- and upper-atmosphere (MU) radar. More recently, Cheong et al. (2008) estimated vertical wind velocities employing an imaging technique and 25-channel system for MU radar. Chen et al. (2008) more specifically investigated the effect of a periodic form of laminar structure possibly generated by Kelvin-Helmholtz instability. Those studies employed imaging techniques taking advantage of multichannel signals to investigate the relationship between scattering structures and wind estimates.

In this paper, we propose a technique that enables us to measure accurate vertical Doppler velocities by adaptively generating a reception beam that is countershifted against an off-vertically shifted echo pattern resulting from aspect-sensitive reflectivity. The technique employs the norm-constrained direction-constrained minimization of power (NC-DCMP) algorithm (Hudson 1981), which was originally developed to stabilize the main and sidelobe behaviors of the basic DCMP sidelobe rejection algorithm (Takao et al. 1976; Capon 1969). The class of NC-DCMP contains the original DCMP. These adaptive beamformers have been used for imaging scatter strength field (Hassenpflug et al. 2008) or canceling clutters (Kamio et al. 2004) in MST radar applications. However, there was no such study that tried to compensate for the angular dependency of the echo patterns to obtain accurate Doppler velocity estimates. We demonstrate the beam adjustment properties of the proposed technique using computer simulation.

The remainder of the paper is outlined as follows. Section 2 describes the simulation model and the procedure for calculating echoes and received signals. Section 3 presents the algorithm for creating the reception beam. Section 4 summarizes our proposition and makes concluding remarks.

\section{Simulation model}

Atmospheric radar measures Doppler velocities of the atmosphere along an effective line-of-sight (LOS) from the radar, which is a moment direction of an echo pattern weighted by an reception beam pattern. For convenience, the product of the echo pattern and reception beam pattern is referred to as the effective echo pattern, which we consider in evaluating the accuracy of vertical wind measurements. In this section, we define the echo pattern and an asymptotic model of the reception signals that is required to determine the reception beam pattern.

The geometrical configuration of the model follows that of the MU radar (Fukao et al. 1985) operated at 
46.5 MHz. The array consists of 475 antennas arranged with uniform triangular spacing at intervals of $4.5 \mathrm{~m}$, which is 0.7 times the wavelength. For simplicity, no explicit element factor or polarization of antennas is considered because these factors are not essential in the following discussions. The first null and one-way halfpower half-width (HPHW) angles for uniform excitation in this configuration are about $3.8^{\circ}$ and $1.8^{\circ}$, respectively, The whole array is divided into 25 groups consisting of 19 antennas each. Each group has its own digital receiver that can store data separately as complex-valued time series or power spectra (Hassenpflug et al. 2008).

Parameters for forming the aspect-sensitive reflectivity pattern in our simulations are adopted from those observed by Worthington et al. (1999), in which Gaussianshaped reflection patterns are exhibited with an HPHW down to $2^{\circ}$ and the off-vertical shift by up to $1^{\circ}$. In the following discussions, these parameters are used as the severest case.

To describe the two-dimensional target space, we use a directional cosine vector, defined as $\boldsymbol{\eta}=\left(\eta_{x}, \eta_{y}\right)^{\mathrm{T}}$, where the superscript $\mathrm{T}$ indicates vector transposition, instead of using the zenith angle $\theta$ and the azimuth angle $\phi$, where

$$
\begin{aligned}
& \eta_{x}=\sin \theta \sin \phi \quad \text { and } \\
& \eta_{y}=\sin \theta \cos \phi
\end{aligned}
$$

for computational efficiency. The target space defined within a region satisfying $\eta_{x}^{2}+\eta_{y}^{2}<1$ is uniformly discretized as a Cartesian grid with an interval $\bar{\eta}$ set to 0.005 for both $\eta_{x}$ and $\eta_{y}$ axes. According to the above definition, each antenna has an implicit element factor $\cos \theta$.

Let $\mathbf{x}_{i}$ be the position vector of the $i$ th antenna, where $\mathbf{x}_{i}=\left(x_{i}, y_{i}\right)^{\mathrm{T}}$. The $z$ component of the antenna position is assumed to be zero. Beam patterns are calculated as follows. Transmission beam pattern $B_{T}(\boldsymbol{\eta})$ is constructed with uniform excitation:

$$
B_{T}(\boldsymbol{\eta})=\mathbf{c}(\boldsymbol{\eta})^{\mathrm{H}} \mathbf{c}\left(\boldsymbol{\eta}_{0}\right)
$$

where the superscript $\mathrm{H}$ denotes the Hermitian transposition,

$$
\mathbf{c}(\boldsymbol{\eta})=\left(\begin{array}{c}
\exp \left[\mathrm{j} 2 \pi \mathbf{x}_{1} \cdot \boldsymbol{\eta}\right] \\
\vdots \\
\exp \left[\mathrm{j} 2 \pi \mathbf{x}_{N} \cdot \boldsymbol{\eta}\right]
\end{array}\right)
$$

and $\boldsymbol{\eta}_{0}$ represents the direction of the transmission beam.

Echo pattern $E(\boldsymbol{\eta})$ is given by

$$
E(\boldsymbol{\eta})=F(\boldsymbol{\eta}) B_{T}(\boldsymbol{\eta})
$$

where $F(\boldsymbol{\eta})$ is an aspect-sensitive reflectivity pattern defined as a Gaussian-shaped function

$$
F(\boldsymbol{\eta})=\exp \left[-h \frac{\left(\boldsymbol{\eta}-\boldsymbol{\eta}_{0}\right)^{2}}{\sigma^{2}}\right],
$$

where $\sigma$ is the HPHW of the function and $h=\log _{e} 2$.

Now we develop the asymptotic uncorrelated signal model for the received echo. This model is constructed assuming that echoes are spatially uncorrelated. With the addition of internal noise, the received signal at the $n$th antenna is written as

$$
r_{n}(t)=\bar{\eta} \sum_{i=1}^{M}\left[a E\left(\boldsymbol{\eta}_{i}\right) s_{i}(t) \exp \left(j 2 \pi \mathbf{x}_{n} \cdot \boldsymbol{\eta}_{i}\right)\right]+v_{n}(t),
$$

where $a$ is the amplitude factor of the echo, $M$ is the number of directions, $s_{i}(t)$ is the echo from the $i$ th direction, and $v_{n}(t)$ is a $\sqrt{1 / 2}$-scaled complex normal random variable. In the following part, $a$ is set to 1.0 , which corresponds to a severe case. The echo $s_{i}(t)$ is uncorrelated from any $s_{j}(t)$ with $j \neq i$ and has normalized power that is $\left\langle\left|s_{i}(t)\right|^{2}\right\rangle=1$, where the angle brackets denote expectation. Let $\mathbf{r}$ be the received signal vector $\left[r_{1}(t), \ldots, r_{N}(t)\right]^{\mathrm{T}}$. The asymptotic covariance matrix is then derived as

$$
\begin{aligned}
\mathbf{R} & =\left\langle\mathbf{r r}^{\mathrm{H}}\right\rangle \\
& =a^{2} \bar{\eta}^{2} \sum_{i=1}^{M}\left\{\left|E\left(\boldsymbol{\eta}_{i}\right)\right|^{2} \mathbf{c}\left(\boldsymbol{\eta}_{i}\right) \mathbf{c}\left(\boldsymbol{\eta}_{i}\right)^{\mathrm{H}}\right\}+\mathbf{I},
\end{aligned}
$$

where I denotes the identity matrix. The reception beam pattern $B_{R}(\boldsymbol{\eta})$ is given by

$$
B_{R}(\boldsymbol{\eta})=\mathbf{c}(\boldsymbol{\eta})^{\mathrm{H}} \mathbf{w}_{\mathrm{NC}}
$$

once an NC-DCMP solution $\mathbf{w}_{\mathrm{NC}}$ is calculated by the algorithm described in the next section. The effective echo pattern $E_{e}(\boldsymbol{\eta})$ is then obtained as

$$
E_{e}(\boldsymbol{\eta})=E(\boldsymbol{\eta}) B_{R}(\boldsymbol{\eta})
$$

Although the covariance matrix is estimated using finite number of samples in real observations, the number of samples is in general large enough to obtain a good estimate of $\mathbf{R}$ in MST applications. For example, the number of time samples to obtain a sample covariance matrix would be 128 , according to a parameter set used for stratospheric and tropospheric observations at the 
MU radar, where the sample is obtained every $64 \mathrm{~m} \mathrm{~s}^{-1}$ for the integration time of $8.192 \mathrm{~s}$, which is a period to estimate a frequency spectrum. The number of 128 is sufficiently larger than the dimension of $\mathbf{R}$ corresponding to the number of signal channels of 25 . However, even this condition is not essential for NC-DCMP because in principle it works with a singular $\mathbf{R}$. This is mentioned in the next section.

The uncorrelated signal model has an advantage that the strength distribution of a signal source agrees with that observed through the reception beams. This property is useful to give an angular distribution of the echo strength field without assuming an unknown spatial mechanism for the creation of the atmospheric echo. A major limitation of the uncorrelated model is that it cannot describe a spatial structure of the wavefront, which may be an issue, for example, in case the echo distribution is split in space in several parts with holding coherence. As far as general narrowbeam observations are concerned, however, the uncorrelated model given the echo pattern viewed from the radar is a valid approximation.

Figure 1a shows a transmission beam pattern pointed at the zenith and the echo pattern obtained from the aspect-sensitive reflection pattern. The aspect sensitivity is assumed as a Gaussian function with a center at $(\theta, \phi)=$ $\left(1.0^{\circ}, 90.0^{\circ}\right)$ and an HPHW of $3^{\circ}$. Note that the echo pattern is clearly shifted off-vertical and skewed owing to the aspect sensitivity although the transmission beam pattern is aligned vertical.

Figure $1 \mathrm{~b}$ shows the conventional fixed (nonadaptive) reception beam pattern and an NC-DCMP beam pattern with NC parameter $\delta$ of 1.5 , which is discussed later. The NC-DCMP reception pattern tilts away from the echo pattern to minimize the total output power under the constant-gain constraint. Note that the NC-DCMP reception beams crosses the fixed beam at $0^{\circ}$, obeying the constant-gain constraint.

Figure 1c shows two effective echo patterns corresponding to the two reception beam patterns in Fig. 1b. Total output signal power is proportional to the area of effective echo patterns, and therefore, the effective echo pattern obtained from NC-DCMP has a smaller area than the other pattern. As a result, the effective echo pattern obtained from NC-DCMP is apparently aligned in the desired vertical direction.

\section{Algorithm for calculating the reception beams}

In this section, we describe the concept and algorithm of NC-DCMP, which is the norm-constrained variation of the original DCMP, and its asymptotic properties in terms of its effects on the effective echo pattern. The principle of NC-DCMP is to find a complex weight
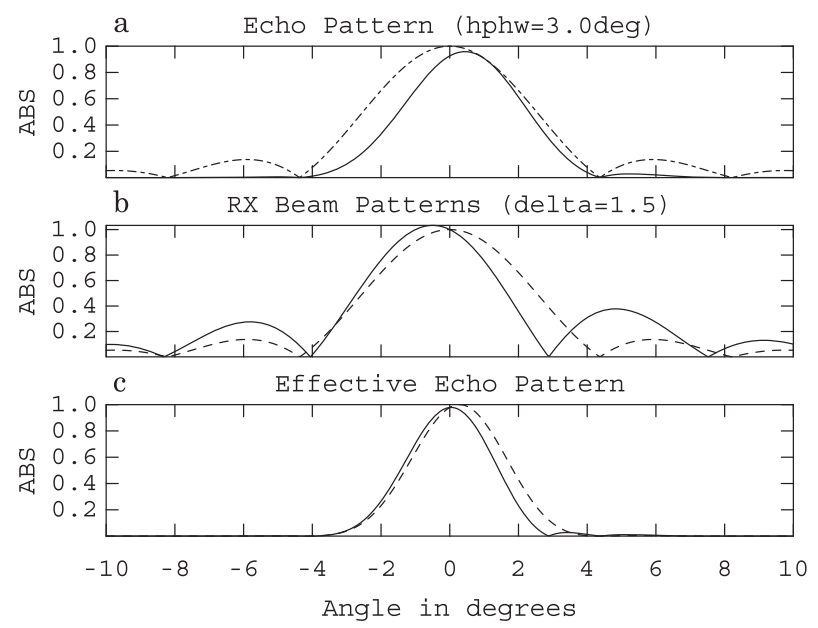

FIG. 1. Simulated patterns based on the MU radar model. The abscissa is the zenith angle $\theta$ in the east-west $\left(\phi=90^{\circ}\right)$ section. (a) Echo pattern (solid) as a product of the transmission pattern (dash-dotted) pointed at the zenith and Gaussian-shaped reflectivity tilted toward $(\theta, \phi)=\left(1.0^{\circ}, 90.0^{\circ}\right)$ with an HPHW of $3.0^{\circ}$ (not shown). (b) The conventional reception beam patterns with fixed uniform excitation (dashed) and the NC-DCMP reception beam pattern (solid), according to the echo pattern shown in (a). (c) Effective echo patterns, which are products of the echo pattern in (a) and either of reception beam in (b), namely, the conventional fixed reception beam (dashed) and the NC-DCMP reception beam (solid).

vector $\mathbf{w}$ for multichannel signals $\mathbf{r}$ such that the average output power $P=\left\langle\left|\mathbf{w}^{\mathrm{H}} \mathbf{r}\right|^{2}\right\rangle$ is minimized, under constantgain and norm constraints regulating the sensitivity toward the desired direction and upper bounds of noise and sidelobe levels, respectively. Let $N$ and $\delta$ be the number of signal sequences and the norm constraint, respectively. The principle is written as

$$
\begin{gathered}
\text { minimize } P=\mathbf{w}^{\mathrm{H}}\left\langle\mathbf{r r}^{\mathrm{H}}\right\rangle \mathbf{w} \\
\text { subject to } \mathbf{c}\left(\boldsymbol{\eta}_{0}\right)^{\mathrm{H}} \mathbf{w}=N \text { and } \quad \mathbf{w}^{\mathrm{H}} \mathbf{w} \leq \delta N .
\end{gathered}
$$

Whereas the constant-gain constraint relates to a specified direction, the signal strength decreases in volume target scenarios as for atmospheric radar, as a consequence of the minimization. In addition to the decrease in signal strength, the resulting weight norm $\mathbf{w}_{\mathrm{NC}}^{\mathrm{H}} \mathbf{w}_{\mathrm{NC}}$ becomes larger than the given quiescent weight norm $\mathbf{c}^{\mathrm{H}} \mathbf{c}$, where these square norms are directly proportional to the output white noise power. Therefore, the algorithm always decreases the signal-to-noise ratio (SNR) compared with a nonadaptive system.

The NC parameter $\delta$, however, defines the upper bound of the norm weights while the original DCMP algorithm is unbound. Hence, the decrease in the SNR due to the minimization principle is guided by the NC parameter $\delta$. 
This problem is classified as convex quadratic optimization and it is guaranteed to have a unique solution. The solution to this problem, denoted $\mathbf{w}_{\mathrm{NC}}$, has the form

$$
\mathbf{w}_{\mathrm{NC}}=\frac{(\mathbf{R}+\lambda \mathbf{I})^{-1} \mathbf{c}}{\mathbf{c}^{\mathrm{H}}(\mathbf{R}+\lambda \mathbf{I})^{-1} \mathbf{c}},
$$

where $\lambda$ gives the magnitude of additional noise so as to suppress the squared norm of $\mathbf{w}_{\mathrm{NC}}$ to less than $\delta N$ (Hudson 1981).

In the case that the DCMP solution $\mathbf{w}_{0}$ satisfies the norm inequality condition $\mathbf{w}_{0}^{\mathrm{H}} \mathbf{w}_{0} \leq \delta N$, obviously $\mathbf{w}_{0}$ is also the NC-DCMP solution, and therefore $\lambda=0$. Otherwise, the NC-DCMP solution $\mathbf{w}_{\mathrm{NC}}$ is bound at

$$
\mathbf{w}_{\mathrm{NC}}^{\mathrm{H}} \mathbf{w}_{\mathrm{NC}}=\delta N .
$$

Unfortunately, in the latter case, there is no simple relationship between $\lambda$ and $\delta$, and the solution should be found with a nonlinear optimization method. However, it is known that the squared norm $\mathbf{w}^{\mathrm{H}} \mathbf{w}$ decreases monotonically as $\lambda$ increases. Therefore, on condition that $\mathbf{R}$ is regular, the solution of $\lambda$ can be found with a simple procedure as follows.

(i) Calculate the DCMP solution $\mathbf{w}_{0}$.

(ii) If $\mathbf{w}_{0}$ satisfies $\mathbf{w}_{0}^{\mathrm{H}} \mathbf{w}_{0} \leq \delta N$, then this $\mathbf{w}_{0}$ is the solution to the NC-DCMP.

(iii) Otherwise, set $\mathbf{w}_{0}$ to the initial value and find $\lambda$ that satisfies (15) by adjusting $\lambda$ with the Newton method.

In case $\mathbf{R}$ is singular, although this is unusual for MST applications, we cannot use the procedure since the solution to DCMP is not defined. The NC-DCMP still have a solution in such a case and we can find it with an ordinary nonlinear search algorithm (e.g., Kamio et al. 2004).

\section{Simulation results and discussions}

To evaluate the goodness of alignment of the effective pattern, we define the effective LOS direction as the peak direction of the effective echo pattern. The effective LOS is plotted versus the offset angle of the Gaussian-shaped reflectivity.

Figure 2 shows the resulting errors of the effective LOS from the vertical when the Gaussian reflectivity, with HPHW of $3.0^{\circ}$, is tilted along the $x$ axis. In the graph, results for $\delta$ of 1.0,1.2, 1.5, 2.0, and $\infty$ (annotated as unbound) are plotted. The $x$ and $y$ components of the errors are separately plotted in the upper and lower panels, respectively. For cases of NC parameter $\delta$ set to

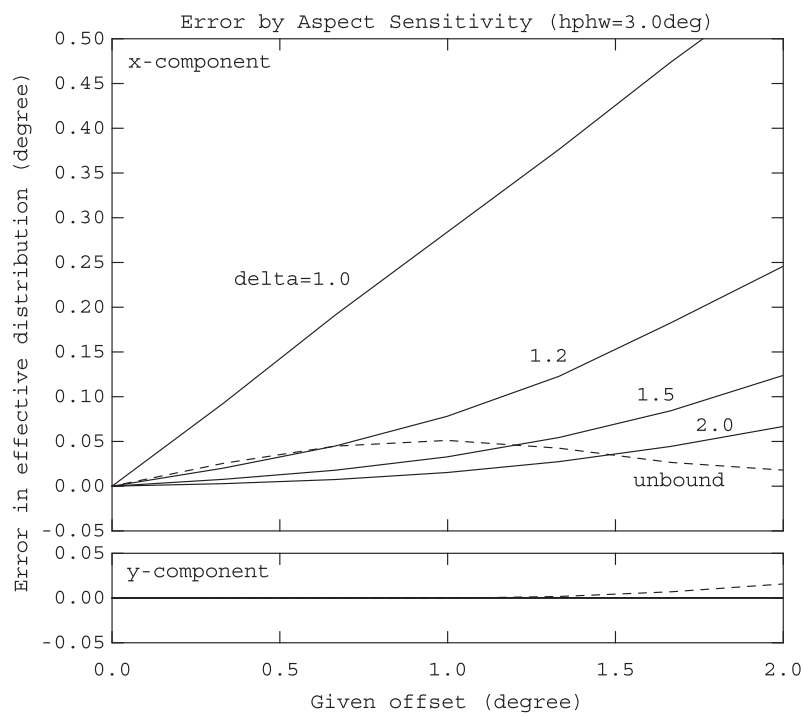

FIG. 2. The two-dimensional error angle of the resulting effective LOS when there is Gaussian-shaped reflectivity with an HPHW of $3^{\circ}$ along the $x$ axis. Resulting errors along the (top) $x$ axis (from the zenith to the east) and (bottom) $y$ axis (from the zenith to the north), respectively, are shown. The number annotated beside each line indicates the NC parameter $\delta$. The $x$ and $y$ axes are scaled in degrees.

1.2, 1.5, and 2.0, the errors are suppressed almost monotonically compared with the case of 1.0 , which is equivalent to the fixed beamformer. In the case of the unbound norm, the error becomes larger in some parts than that in bound cases although the SNR becomes worse. It should also be noted that the reflectivity offset is always given along the $x$ axis and this has little effect on the orthogonal component of the effective LOS error except in the case of the unbound norm.

In the case of the HPHW being $3.0^{\circ}$, the resulting $x$ components of the errors in the effective LOS are $0.284^{\circ}$, $0.078^{\circ}, 0.033^{\circ}$, and $0.015^{\circ}$, respectively, for $\delta$ of $1.0,1.2$, 1.5 , and 2.0, at a tilt angle of $1.0^{\circ}$. Hence, in the case that a decrease in the SNR is acceptable to $1 \mathrm{~dB}(\sim 1.26)$, the error in the LOS is suppressed to less than $0.1^{\circ}$. If the worsening of the SNR is acceptable to about $3 \mathrm{~dB}$ $(\sim 2.0)$, which is often the case for tropospheric and lower-stratospheric observations using vertical beams for which usually much larger SNR is obtained than for oblique beams with MST radar, the error in LOS is suppressed to nearly $1 / 20$ of that in the fixed case in this condition.

To evaluate severest cases, Fig. 3 shows the errors in LOS under the condition of an HPHW of $2.0^{\circ}$. The $x$ components of the errors are $0.474^{\circ}, 0.226^{\circ}, 0.112^{\circ}$, and $0.042^{\circ}$, respectively, for $\delta$ of $1.0,1.2,1.5$, and 2.0 , at a tilt angle of $1.0^{\circ}$. Clearly, these data are worse than those with an HPHW of $3.0^{\circ}$. Even in this severe condition, 


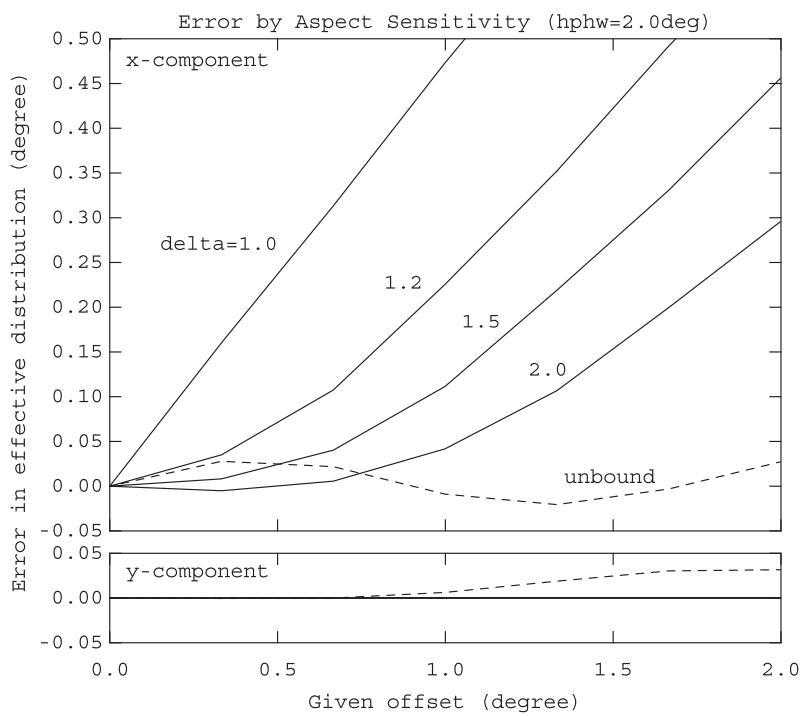

FIG. 3. As in Fig. 2, but with Gaussian-shaped reflectivity with an HPHW of $2^{\circ}$.

however, the errors in the cases of $\delta$ of 1.5 and 2.0 approach $0.1^{\circ}$ and less than $0.05^{\circ}$, respectively.

The coefficient of contamination from horizontal wind components to the vertical components is given by the sine of LOS error angle, which is $1.745 \times 10^{-3}$ and $0.873 \times 10^{-3}$, respectively, for off-vertical angles of $0.1^{\circ}$ and $0.05^{\circ}$. For horizontal wind of $30 \mathrm{~m} \mathrm{~s}^{-1}$, these factors induce contaminations of the vertical components of 52.35 and $26.19 \mathrm{~mm} \mathrm{~s}^{-1}$ and they are roughly $1 / 20$ and $1 / 10$, respectively, of the values for a conventional fixed beamformer.

In real observations, some parameters that are ignored in this study such as the antenna element factor, stochastic fluctuation, background noise level, and correlated parts of echoes should quantitatively have some effect on the results. Hence the "best" parameter setting should be identified based on observed data. However, evaluating the new technique that provides accuracy of measurement needs a self-consistent scheme. This requires a statistical analysis on a sufficient dataset, which is an issue for a future work.

\section{Conclusions}

The present study introduced a technique to control the beam pattern that enables accurate measurement of the vertical velocity of wind. By choosing an appropriate NC parameter, the method can adjust the reception beam pattern while suppressing the sidelobe and noise levels at a designated threshold. The norm constraint is essential for this technique in order to control the balance between the final error of the LOS and the SNR.
With an NC parameter of $\delta<2.0$, where the loss in SNR of roughly $3 \mathrm{~dB}$ is allowed compared to that of the conventional beamformer, the errors in effective LOS are $0.015^{\circ}$ and $0.042^{\circ}$, respectively, for HPHWs of $3.0^{\circ}$ and $2.0^{\circ}$ when the reflectivity pattern is shifted by $1.0^{\circ}$. These errors are approximately $1 / 20$ and $1 / 10$ of those of the conventional fixed beamformer.

Although the present paper focused on the vertical wind measurement, the proposed technique itself can be applied to oblique beams. The well-known problem of systematic measurement bias for oblique beams, owing to the offset of the scatterer within a volume in the case of a laminar turbulent layer (Fukao et al. 1988), should be mitigated with the proposed technique. Thus, the technique is also useful for accurate Doppler measurement with any angled beam.

\section{REFERENCES}

Alexander, S. P., A. R. Klecociuk, M. C. Pitts, A. J. McDonald, and A. Arevalo, 2011: The effect of orographic gravity waves on antarctic polar stratospheric cloud occurrence and composition. J. Geophys. Res., 116, D06109, doi:10.1029/2010JD015184.

Capon, J., 1969: High-resolution frequency-wavenumber spectrum analysis. Proc. IEEE, 57, 1408-1418.

Chen, J. S., G. Hassenpflug, and M. Yamamoto, 2008: Tilted refractive-index layers possibly caused by Kelvin-Helmholtz instability and their effects on the mean vertical wind observed with multiple-receiver and multiple-frequency imaging techniques. Radio Sci., 43, RS4020, doi:10.1029/2007RS003816.

Cheong, B. L., T. Y. Yu, R. D. Palmer, K. F. Yang, M. W. Hoffman, S. J. Frasier, and F. J. Lopez-Dekker, 2008: Effects of wind field inhomogeneities on doppler beam swinging revealed by an imaging radar. J. Atmos. Oceanic Technol., 25, 1414 1422.

Eckermann, S. D., L. Hoffman, M. Höpfner, D. L. Wu, and M. J. Alexander, 2009: Antarctic NAT PSC belt of June 2003: Observational validation of the mountain wave seeding hypothesis. Geophys. Res. Lett., 36, L02807, doi:10.1029/2008GL036629.

Fukao, S., T. Sato, T. Tsuda, S. Kato, K. Wakasugi, and T. Makihira, 1985: The MU radar with an active phased array system: 1. Antenna and power amplifiers. Radio Sci., 20, 11551168.

,-- , P. T. May, T. Tsuda, S. Kato, M. Inaba, and I. Kimura, 1988: A systematic error in MST/ST radar wind measurement induced by a finite range volume effect: 1 . Observational results. Radio Sci., 23, 59-73.

—, M. F. Larsen, M. D. Yamanaka, H. Furukawa, T. Tsuda, and S. Kato, 1991: Observations of a reversal in long-term average vertical velocities near the jet stream wind maximum. Mon. Wea. Rev., 119, 1479-1489.

Gage, K. S., and J. L. Green, 1978: Evidence for specular reflection from monostatic VHF radar observations of the stratosphere. Radio Sci., 13, 991-1001.

Hassenpflug, G., M. Yamamoto, H. Luce, and S. Fukao, 2008: Description and demonstration of the new middle and upper atmosphere radar imaging system: 1-d, 2-d and 3-d imaging of troposphere and stratosphere. Radio Sci., 43, RS2013, doi:10.1029/2006RS003603. 
Hudson, J. E., 1981: Adaptive Array Principles. Peter Peregrinus, $175 \mathrm{pp}$.

Kamio, K., K. Nishimura, and T. Sato, 2004: Adaptive sidelobe control for clutter rejection of atmospheric radars. Ann. Geophys., 22, 4005-4012.

Kohma, M., and K. Sato, 2011: The effects of atmospheric waves on the amounts of polar stratospheric clouds. Atmos. Chem. Phys., 11, 11 535-11 552.

McLandress, C., and T. G. Shepherd, 2009: Simulated anthropogenic changes in the Brewer-Dobson circulation including its extension to high latitudes. J. Climate, 22, 1516-1540.

Muschinski, A., 1996: Possible effect of Kelvin-Helmholtz instability on VHF radar observations of the mean vertical wind. J. Appl. Meteor., 35, 2210-2217.

Nastrom, G. D., and T. E. VanZandt, 1994: Mean vertical motions seen by radar wind profilers. J. Appl. Meteor., 33, 984-995.

Okamoto, K., K. Sato, and H. Akiyoshi, 2011: A study on the formation and trend of the Brewer-Dobson circulation. J. Geophys. Res., 116, D10117, doi:10.1029/2010JD014953.

Palmer, R. D., M. F. Larsen, R. F. Woodman, S. Fukao, M. Yamamoto, T. Tsuda, and S. Kato, 1991: VHF radar interferometry measurements of vertical velocity and the effect of tilted refractivity surfaces on standard doppler measurements. Radio Sci., 26, 417-427.

Plumb, R. A., 2002: Stratospheric transport. J. Meteor. Soc. Japan, 80, 793-809.

Röttger, J., 1980: Reflection and scattering of VHF radar signals from atmospheric refractivity structures. Radio Sci., 15, $259-276$.
Sato, K., 1990: Vertical wind disturbances in the troposphere and lower stratosphere observed by the MU radar. J. Atmos. Sci., 47, 2803-2817.

_ 1993: Small-scale wind disturbances observed by the MU radar during the passage of Typhoon Kelly. J. Atmos. Sci., 50, 518-537.

Sato, T., N. Ao, M. Yamamoto, S. Fukao, T. Tsuda, and S. Kato, 1991: A typhoon observed with the MU radar. Mon. Wea. Rev., 119, 755-768.

Takao, K., M. Fujita, and N. Kikuma, 1976: An adaptive antenna array under directional constraint. IEEE Trans. Antennas Propag., AP-24, 662-669.

Takayabu, I., M. D. Yamanaka, and S. Fukao, 2000: A mechamism of the reversal of long-term average velocities around East Asia during the cold season. J. Meteor. Soc. Japan, 78, 13-23.

Taylor, M. J., and Coauthors, 2011: High-latitude gravity wave measurements in noctilucent clouds and polar mesospheric clouds. Aeron. Earth's Atmos. Ionos., 2, 93-105, doi:10.1007/ 978-94-007-0326-1_7.

Tsuda, T., T. Sato, K. Hirose, S. Fukao, and S. Kato, 1986: MU radar observations of the aspect sensitivity of backscattered VHF echo power in the troposphere and lower stratosphere. Radio Sci., 21, 971-980.

_ , T. E. VanZandt, and H. Saito, 1997: Zenith-angle dependence of VHF specular reflection echoes in the lower atmospere. J. Atmos. Sol. Terr. Phys., 59, 761-775.

Worthington, R., R. Palmer, and S. Fukao, 1999: Complete maps of the aspect sensitivity of VHF atmospheric radar echoes. Ann. Geophys., 17, 1116-1119. 